\title{
RESPOSTA DAS VIDEIRAS A DIFERENTES MODOS DE DISTRIBUIÇÃO DE COMPOSTO ORGÂNICO NO SOLO ${ }^{1}$
}

\author{
GEORGE WELLINGTON BASTOS DE MELO², GUSTAVO BRUNETTO², \\ ALEX BASSO $^{4}$, JANAINA HEINZEN ${ }^{5}$
}

RESUMO - O trabalho objetivou avaliar o estado nutricional, a produção e a composição do mosto de uvas em videiras submetidas a diferentes modos de distribuição de composto orgânico no solo. O experimento foi conduzido em um vinhedo da cv. Cabernet Sauvignon, com plantas enxertadas sobre o porta-enxerto 'Paulsen 1103 ' em um solo Neossolo Litólico, em Bento Gonçalves (RS). Em agosto de 2006, foram implantados os tratamentos (T1) testemunha; (T2) aplicação de composto orgânico na superfície do solo sempre no mesmo lado da linha de plantio; (T3) aplicação de composto em lados alternados da linha de plantio ao longo dos anos; (T4) aplicação de composto em covas sempre do mesmo lado da linha de plantio, e (T5) aplicação de composto em covas em lados alternados na linha de plantio ao longo dos anos. Nas safras de 2006/2007, 2007/2008 e 2008/2009, os ramos da poda de inverno e o tecido da poda de verão foram secos e determinada a produção de matéria seca. Folhas completas foram coletadas, secas, moídas e submetidas à análise do teor total de N, P, K, Ca e Mg. Na maturação, foi contado o número de cachos e pesados, e, em seguida, bagas foram coletadas e determinados os valores de sólidos solúveis, $\mathrm{pH}$, acidez titulável, ácido tartárico e málico. A distribuição de composto em covas sempre do mesmo lado da linha de plantio ou em lados alternados ao longo dos anos aumentou a quantidade de matéria seca dos ramos podados no inverno, especialmente, nas primeiras safras depois de sua aplicação. A aplicação e o modo de distribuição do composto na superfície do solo ou incorporado, anualmente no mesmo lado da linha de plantio ou alternado entre os lados ao longo dos anos, não afetaram a produção de uva, o estado nutricional e a composição do mosto, e, quando necessário, o composto orgânico deve ser aplicado na superfície do solo sem incorporação, em um mesmo lado da linha de plantio ou alternado entre os lados ao longo dos anos.

Termos para indexação: Adubação orgânica, nutrição mineral, qualidade da uva, Vitis vinifera.

\section{RESPONSE OF THE GRAPEVINES TO DIFFERENT FORMS OF DISTRIBUTION OF ORGANIC COMPOST IN SOIL}

\begin{abstract}
The experiment aimed to evaluate leaves nutrient content, yield and must composition in grapevines, which were submitted to the application to different forms of distribution of organic compost on the vineyard soil with Cabernet Sauvignon grapevine, grafted on 'Paulsen 1103' rootstock, at Udorthent soil, in the Southern Brazil, Bento Gonçalves city. The treatments were (T1) without organic compost; (T2) organic compost application with distribution on the soil surface, always at same side of the plant rows; (T3) organic compost application with distribution on alternate sides of the plant rows; (T4) organic compost application in holes always on the same side of the plant rows and (T5) organic compost application in holes on alternate sides of the plant rows. The weight of pruning was evaluated during winter and summer. Leaves were collected, oven-dried, weighted and total N, P, K, Ca and Mg contents were analyzed. In the harvest, the bunch number and weight, grape yield and $\mathrm{pH}$, soluble solids, acidity, tartaric and malic acids in the must was evaluated. The compost distribution in holes always on the same side or in alternated sides of the plant rows, along of the years, increased the amount of dry matter of the branches pruned in the winter, especially, in the first years after your application. The application and forms of compost distribution in the soil surface or incorporated, annually on the same side of the rows or alternate sides of the plants, along the years, do not affected the yield grape, nutrient content of leaves and must composition. The organic compost should, when necessary, should be applied in the soil surface without incorporation, on the same side of the plant rows or on alternate sides of the plants row.

Index terms: organic fertilization, mineral nutrition, Vitis vinifera, grape quality, Cabernet Sauvignon.
\end{abstract}

${ }^{1}$ (Trabalho 185-11). Recebido em: 27-06-2011. Aceito para publicação em: 30-05-2012. Apoio financeiro: CNPq e Embrapa Uva e Vinho. ${ }^{2}$ Enge. Agr., Dr. em Ciência do Solo, Pesquisador Embrapa Uva e Vinho, e-mail: george@cnpuv.embrapa.br.

${ }^{3}$ Eng. Agr., Dr. em Ciência do Solo, Prof. Depto. de Eng. Agrícola e do Programa de Pós-Graduação em Agroecossistemas da -UFSC/ CCA. Florianópolis-SC, Brasil, e-mail: brunetto.gustavo@gmail.com

${ }^{4}$ Acadêmico do Curso de Graduação em Engenharia de Biossistemas e Biotecnologia da UERGS, e-mail: alex.basso54@hotmail.com ${ }^{5}$ Acadêmico do Curso de Graduação em Agronomia da Universidade Federal de Santa Catarina (UFSC), e-mail: heinzen6@hotmail.com 


\section{INTRODUÇÃO}

As fontes de adubo orgânico, como o composto orgânico, têm sido usadas na adubação de crescimento e de manutenção em vinhedos com uvas de mesa. No entanto, nos últimos anos, o uso delas tem-se intensificado na adubação de manutenção em vinhedos de viníferas, especialmente em sistemas de produção orgânica, como os realizados na Serra Gaúcha do Rio Grande do Sul (RS), que é a mais importante região vitivinícola do Brasil.

O composto orgânico pode ser obtido pela compostagem aeróbia de resíduos, como a borra de suco, o engaço de uva, a serragem, entre outros materiais (BERTRAN et al., 2004), e, quando aplicado em solos cultivados com videira, especialmente, naqueles com histórico de degradação, pode, ao longo dos anos, aumentar o teor de carbono orgânico total e, por consequência, a matéria orgânica do solo (BUSTAMANTE et al., 2011), e melhorar a porosidade (PIAMONTI et al., 1995; PIAMONTI, 1998), bem como a estrutura do solo (GOULET et al., 2004). Além disso, para a videira, o composto tem sido usado como fonte de nutrientes, entre eles, o nitrogênio $(\mathrm{N})$, o fósforo $(\mathrm{P})$ e o potássio $(\mathrm{K})$ (KORBOULEWSKY et al., 2002; CHISTOU et al., 2006; RAMOS; MARTÍNEZ-CASASNOVAS, 2006; NEIDEL et al., 2007).

Nos vinhedos da região Sul do Brasil, não se conhecem a necessidade nem o melhor modo de distribuição do composto orgânico no solo. Por isso, alguns vitivinicultores distribuem o composto no interior de covas abertas no mesmo lado das linhas de plantio das videiras ou em lados alternados ao longo dos anos e, com isso, espera-se maior área de contato do solo com o composto, estimulando sua decomposição pelos microrganismos do solo e, por consequência, a liberação de nutrientes, especialmente nitrogênio $(\mathrm{N})$, fósforo $(\mathrm{P}) \mathrm{e}$ potássio (K). No entanto, a abertura de covas provoca o corte de raízes, o que potencializa a ocorrência de doenças, como a fusariose (Fusarium oxysporum f.sp. herbemontis) e o pé-preto (Cylindrocarpon destructians) (SANHUEZA; SÔNEGO, 1993), o que diminui o acúmulo de substâncias de reservas nas raízes, como as nitrogenadas e os carboidratos, determinantes no desenvolvimento das plantas no próximo ciclo (SCHENATO et al., 2007). Como opção à aplicação do composto em covas, ele pode ser adicionado na superfície do solo, do mesmo lado da linha de plantio ou em lados alternados ao longo dos anos, sendo que, nesta situação, haverá menor área de contato do adubo orgânico com a superfície do solo, o que pode diminuir sua taxa de decompo- sição e a liberação de nutrientes. Além disso, o custo de aplicação é reduzido, bem como a incidência de doenças radiculares e, com isso, espera-se maior vida útil das videiras. No entanto, a aplicação, bem como o modo de distribuição podem causar impacto no estado nutricional das videiras e até na produção, como relatado por Pinamonti (1998), em videiras submetidas à aplicação de fontes de adubo orgânico na Itália e por Morlat (2008), em vinhedos na França, e algumas vezes até sobre a composição da uva e do seu mosto, como comentado por Morlat e Symoneaux (2008), aplicando adubo orgânico em videiras na França e por Nash et al. (2011), em vinhedos na Austrália. Porém, nas condições edafoclimáticas da Serra Gaúcha essas informações são escassas ou inexistentes.

O presente trabalho teve como objetivo avaliar o estado nutricional, a produção e a composição do mosto de uvas em videiras submetidas a diferentes modos de distribuição de composto orgânico no solo.

\section{MATERIAL E MÉTODOS}

O trabalho foi conduzido na área experimental da Embrapa Uva e Vinho, localizada no município de Bento Gonçalves (Latitude 29 09’ 44” S e Longitude $51^{\circ} 31^{\prime} 50^{\prime}$ 'W), região da Serra Gaúcha do Rio Grande do Sul, Brasil. O vinhedo experimental foi instalado em setembro de 2005, utilizando-se de mudas da cv. Cabernet Sauvignon, enxertadas sobre o porta-enxerto 'Paulsen 1103'. As videiras foram plantadas na densidade de 3.333 plantas por hectare (2,5 $\mathrm{m} \times 1,20 \mathrm{~m})$ e conduzidas em espaldeira. O solo foi classificado como Neossolo Litólico (EMBRAPA, 2006) (Tabela 1). O clima da região, segundo a classificação de Köeppen é do tipo subtropical Cfa, sendo os meses mais frios junho e julho, com temperaturas médias mínimas de $8^{\circ} \mathrm{C}$ e médias máximas de $17^{\circ} \mathrm{C}$. Os meses mais quentes são janeiro e fevereiro, com temperaturas médias mínimas de $17^{\circ} \mathrm{C}$ e médias máximas de $26^{\circ} \mathrm{C}$ (BRUNETTO, 2008). Os dados climáticos obtidos durante a condução do experimento estão apresentados na Tabela 2.

Em julho de 2005, foram aplicados na superfície do solo, com posterior incorporação na camada de $0-20 \mathrm{~cm}, 1.800 \mathrm{~kg} \mathrm{ha}^{-1}$ de calcário dolomítico para elevar o $\mathrm{pH}$ em água até 6,0 (CQFS-RS/SC, 2004). Em agosto de 2006, foram implantados os tratamentos: T1- Testemunha sem aplicação de composto orgânico; T2- aplicação anual de $20 \mathrm{~m}^{3}$ ha $^{-1}$ de composto orgânico, 15 dias antes da poda de inverno, em faixa de $30 \times 60 \mathrm{~cm}$, na superfície do solo (aproximadamente a $30 \mathrm{~cm}$ do caule da planta) e sempre do mesmo lado da linha de plantio; T3- Idem 
o T2, mas o composto foi aplicado anualmente, em lados alternados da linha de plantio das plantas; T4Idem o T2, mas o composto foi distribuído em covas (dimensões $20 \times 60 \times 20 \mathrm{~cm}$ ), na linha de plantio, sempre do mesmo lado das plantas, e T5- Idem o T2, mas o composto orgânico foi aplicado em covas (dimensões $20 \times 60 \times 20 \mathrm{~cm}$ ) e com distribuição anual em lados alternados da linha de plantio. Nos tratamentos onde foram abertas covas, o solo retirado delas foi reservado e, após a adição do composto no interior das covas, ele foi depositado sobre o composto. A dose de $20 \mathrm{~m}^{3}$ ha $^{-1}$ de composto orgânico foi aplicada conforme recomendação técnica para solos com teor médio de matéria orgânica $(26,0-50,0$ $\mathrm{g} \mathrm{kg}^{-1}$ ) (CQFS-RS/SC, 2004). O composto aplicado ao longo dos anos de condução do experimento foi produzido a partir de resíduos de agroindústrias de suco, como borra e engaço, bem como de serragem (Tabela 3). O delineamento experimental usado foi blocos ao acaso, com três repetições, e cada parcela foi formada por cinco plantas, distribuídas ao longo da linha de plantio, sendo avaliadas as três plantas centrais.

As videiras, durante a condução do experimento, receberam a aplicação de fungicidas e inseticidas, seguindo recomendações técnicas para a cultura, bem como a uma poda seca de inverno e uma poda verde, como proposto por Mandelli e Miele (2008). Durante o período vegetativo e produtivo das plantas, em cada safra, a linha de plantio das plantas foi mantida isenta de plantas daninhas, usando herbicida. Já nas entrelinhas das videiras, foram mantidas plantas espontâneas, como o picão-preto (Bidens pilosa L.), o papuã (Brachiaria plantaginia), a grama-seda (Cynodon dactylon), o milhã (Digitalia sanguinalis) e a serralha (Sonchus oleraceus) e, quando necessário, realizaram-se roçadas. Nas safras de 2006/2007, $2007 / 2008$ e 2008/2009, na plena floração das videiras, foram coletadas folhas completas localizadas na posição oposta ao cacho. Em seguida, elas foram secas em estufa com ar forçado, à temperatura de $60^{\circ} \mathrm{C}$, até matéria seca constante. Posteriormente, elas foram preparadas e submetidas à análise dos teores totais de $\mathrm{N}, \mathrm{P}, \mathrm{K}$, cálcio $(\mathrm{Ca})$ e magnésio $(\mathrm{Mg})$ (TEDESCO et al., 1995). As videiras, em cada safra e no mês de outubro, foram submetidas a uma poda verde, e o material foi reservado. Também, no mês de julho, as videiras foram submetidas à poda de inverno, tipo mista, mantendo-se esporões e varas com sete gemas por planta. Os ramos podados foram coletados e reservados. O material da poda verde e os ramos da poda de inverno foram secos em estufa com ar forçado, à temperatura de $60^{\circ} \mathrm{C}$, até matéria seca constante e, em seguida, pesados. Nas três safras, na maturação das uvas, quando atingiram $17^{\circ}$ Brix, foi contado o número de cachos por planta. Logo depois, foram coletados quatro cachos no centro da planta e quatro na parte externa e, em seguida, pesados. Posteriormente, foram coletadas bagas na parte inferior, mediana e superior de cada cacho e reservadas. Em seguida, os cachos restantes nas plantas foram colhidos e pesados. Posteriormente, as bagas de uvas reservadas foram amassadas e, a partir do mosto, foram realizadas as seguintes análises: teor de sólidos solúveis (SS), usando refratômetro digital de bancada com autocompensação de temperatura; $\mathrm{pH}$, com potenciômetro digital; acidez titulável (AT) por titulação com $\mathrm{NaOH} 0,1 \mathrm{~N}$, usando o azul de bromotimol como indicador, e ácido tartárico e málico, por cromatografia líquida de alta eficiência (AUGUSTE, 1979).

Os resultados obtidos foram submetidos à análise de variância e, quando significativos, as médias foram submetidas ao teste de comparação de médias Tukey, a 5\% de probabilidade. As análises estatísticas foram realizadas utilizado o software SAS (SAS, 2008).

\section{RESULTADOS E DISCUSSÃO}

\section{Produção de matéria seca da poda e compo- nentes de produção}

A maior produção de matéria seca da poda de inverno na safra de 2006/2007 foi encontrada no tratamento com a aplicação anual de $20 \mathrm{~m}^{3} \mathrm{ha}^{-1} \mathrm{de}$ composto orgânico, 15 dias antes da poda de inverno, em covas e em lados alternados da linha de plantio ao longo dos anos (T5) (Tabela 4). Já na safra de 2007/2008, a maior produção de matéria seca da poda de inverno foi observada no $\mathrm{T} 5$ e no tratamento onde o composto foi aplicado em covas abertas na linha de plantio, sempre do mesmo lado das plantas (T4). Nessas duas safras (2006/2007 e 2007/2008), a produção de matéria seca da poda de inverno obtida nos tratamentos quando os $20 \mathrm{~m}^{3} \mathrm{ha}^{-1}$ ano $^{-1}$ de composto orgânico foram aplicados em faixa de 30 x $60 \mathrm{~cm}$ na superfície do solo, sempre do mesmo lado da linha de plantio (T2) e em lados alternados da linha de plantio ao longo dos anos (T3) foi menor que as encontradas nos tratamentos T4 e T5, quando o composto foi incorporado, porém foram maiores que as obtidas no tratamento sem a aplicação de composto. No entanto, a produção de matéria seca da poda de inverno, na safra de 2008/2009, foi maior no tratamento $\mathrm{T} 3$.

Com base nesses resultados, verifica-se primeiro que as videiras, mesmo cultivadas em um solo com 29,0 $\mathrm{g} \mathrm{kg}^{-1}$ (Tabela 1), interpretado como 
médio (26,0-50,0 $\mathrm{g} \mathrm{kg}^{-1}$ ) (CQFS-RS/SC, 2004); com $14,2 \mathrm{mg} \mathrm{dm}^{-3}$ de P disponível, interpretado como alto (12,1-24, $0 \mathrm{mg} \mathrm{kg}^{-1}$, para solos classe $3=40-21 \%$ de argila) (CQFS-RS/SC, 2004) e com $145 \mathrm{mg} \mathrm{dm}^{-3} \mathrm{de}$ $\mathrm{K}$ trocável, interpretado como alto (91-180 $\mathrm{mg} \mathrm{dm}^{-3}$, para solos com $\mathrm{CTC}_{\mathrm{pH} 7,0} 17,17 \mathrm{cmol}_{\mathrm{c}} \mathrm{kg}^{-1}$ ), aumentaram a produção de ramos podados no inverno com a aplicação de $20 \mathrm{~m}^{3} \mathrm{ha}^{-1}$ ano $^{-1}$ de composto incorporado em covas no solo ou aplicado na sua superfície, no mesmo lado da linha de plantio ou em lados alternados ao longo dos anos, comparativamente à testemunha, que não recebeu a aplicação de composto (Tabela 4). Mas, as maiores produções de ramos podados no inverno, na safra de 2006/2007, foi observada no tratamento T5 e, na safra de 2007/2008, nos tratamentos T4 e T5. Em ambos os tratamentos, o composto foi incorporado, e o incremento da produção de ramos, possivelmente, pode ser atribuído à maior área de contato do solo com o composto orgânico, o que estimula sua decomposição pelos microrganismos e, por consequência, a liberação de nutrientes, especialmente o $\mathrm{N}$, que pode ser absorvido pelas videiras e estimular o crescimento vegetativo (PIAMONTI, 1998). Porém, na terceira safra avaliada (2008/2009), a maior produção de matéria seca da poda de inverno e também da poda verde de verão (Tabela 4) foi encontrada nas videiras submetidas à aplicação superficial de composto, em especial quando ele foi aplicado em lados alternados da linha de plantio, o que pode ser atribuído a sua menor área de contato com a superfície do solo, o que retarda a atividade da biomassa microbiana e a sua decomposição e, por consequência, prolonga a liberação de nutrientes (KORBOULEWSKY et al., 2002; CHISTOU et al., 2006; NEIDEL et al., 2007), podendo aumentar o sincronismo entre a liberação e a absorção de nutrientes pela planta.

Convém salientar que, na safra de 2008/2009, em todos os tratamentos, os valores de matéria seca da poda verde foram maiores que os encontrados em todos os tratamentos nas safras anteriores (2006/2007 e 2007/2008) (Tabela 4), e isso pode ser explicado em parte pelos maiores volumes de precipitações nos meses de agosto e setembro, que antecederam a poda verde (Tabela 2). Isso pode, provavelmente, ter aumentado a disponibilidade de água no solo e contribuído para a maior absorção de nutrientes, como o N, P e Mg, cujos teores totais na folhas, em todos os tratamentos, foram maiores na safra de 2008/2009, comparativamente às demais safras (Tabela 5), estimulando o crescimento vegetativo da parte aérea.

O maior número de cachos por planta, na safra de 2006/2007, foi encontrado quando o com- posto orgânico foi aplicado na superfície do solo (T2 e T3), mas nas demais safras (2007/2008 e 2008/2009) a variável não foi afetada pela aplicação nem pelo modo de distribuição do composto, o que concorda com os dados de peso médio dos cachos e produção de uva por hectare, obtidos nas três safras avaliadas (Tabela 4). Esses dados concordam com os obtidos por Brunetto et al. (2006; 2007; 2008; 2009 e 2011), que relatam a pouca interferência de adubações sobre a produção de uva de viníferas, o que segundo os autores pode ser atribuído aos teores de nutrientes no solo, como os de $\mathrm{P}$ disponível e $\mathrm{K}$ trocável, que no presente trabalho foram altos, mas também aos teores de matéria orgânica, que, somados à decomposição de resíduos (de poda, folhas senescentes e resíduos de plantas de cobertura), podem conferir boa disponibilidade de $\mathrm{N}$ para as videiras. No entanto, convém destacar que experimentos com adubação orgânica devem ser realizados por um período mais longo, para verificar o real impacto dela sobre os componentes de produção, por exemplo, o número de cachos por planta, ou mesmo sobre a produção de uva.

\section{Estado nutricional e composição do mosto}

A aplicação de composto, bem como os modos de distribuição no solo não afetaram os teores totais de $\mathrm{N}, \mathrm{P}$ e $\mathrm{Mg}$ nas folhas completas de videira coletadas no florescimento, nas safras de 2006/2007, $2007 / 2008$ e 2008/2009, e os totais de K e Ca nas safras de 2007/2008 e 2008/2009 (Tabela 5). Porém, na safra de 2006/2007, os maiores teores totais de K foram encontrados no tratamento com a aplicação do composto incorporado em covas na linha de plantio (T4), mas os valores não diferiram dos encontrados nos tratamentos com a aplicação do composto na superfície, sempre no mesmo lado da linha de plantio (T2) e quando da aplicação dele em covas em lados alternados da linha de plantio (T5). Nesta mesma safra, os teores totais de Ca foram maiores nos tratamentos com a aplicação do composto incorporado em covas na linha de plantio, no mesmo lado das plantas (T3) e em lados alternados (T5).

A pouca interferência, na maioria das safras, da aplicação e do modo de distribuição do composto sobre o teor total de nutrientes nas folhas concorda com os dados obtidos por Morlat (2008), que relata que a aplicação de dejeto bovino como fonte de nutrientes para a videira não aumenta o teor de nutrientes na folha completa até seis anos depois do início de sua aplicação, mas aumenta depois de onze anos. Da mesma forma, Pinamonti (1998) também não verificou efeitos significantes da aplicação de composto no solo sobre os teores de nutrientes nas folhas de videiras cultivadas na Itália.

Ressalta-se que os teores totais de $\mathrm{N}$ e $\mathrm{P}$ nas folhas completas, coletadas nas videiras em todos os tratamentos, nas safras de 2006/2007 e 2007/2008, 
foram normais $\left(\mathrm{N}=16-24 \mathrm{~g} \mathrm{~kg}^{-1}\right.$ e $\mathrm{P}=1,2-4,0 \mathrm{~g}$ $\left.\mathrm{kg}^{-1}\right)$ (CQFS-RS/SC, 2004) e acima do normal na safra de 2008/2009 $\left(\mathrm{N}=>24 \mathrm{~g} \mathrm{~kg}^{-1}\right.$ e $\mathrm{P}=>4,0 \mathrm{~g}$ $\left.\mathrm{kg}^{-1}\right)$ (CQFS-RS/SC, 2004). Para os teores totais de $\mathrm{K}$ nas folhas completas, coletadas nas videiras na maioria dos tratamentos, ao longo das três safras, foram normais $\left(\mathrm{K}=8-16 \mathrm{~g} \mathrm{~kg}^{-1}\right)(\mathrm{CQFS}-\mathrm{RS} / \mathrm{SC}$, 2004), sendo o teor total de Ca nas folhas completas, nas duas primeiras safras (2006/2007 e 2007/2008), normal (16-24 $\left.\mathrm{g} \mathrm{kg}^{-1}\right)(\mathrm{CQFS}-\mathrm{RS} / \mathrm{SC}, 2004)$, mas na safra de 2008/2009, abaixo do normal $\left(<16 \mathrm{~g} \mathrm{~kg}^{-1}\right)$. Por outro lado, os teores totais de $\mathrm{Mg}$ nas folhas completas, em todos os tratamentos, foram normais ou muito próximos do normal $\left(>2-6 \mathrm{~g} \mathrm{~kg}^{-1}\right.$ ) (Tabela Os valores de $\mathrm{SS}, \mathrm{pH}$, acidez titulável, ácido málico e tartárico do mosto das uvas de videiras não foram afetados pela aplicação ou modo de distribuição do composto orgânico (Tabela 6), corroborando os dados de produção (Tabela 4) e a maioria dos teores totais de nutrientes nas folhas completas obtidos nas três safras (Tabela 5). Esses resultados concordam com os obtidos por Nash et al. (2011), que relataram que a aplicação de biossólido no solo cultivado com a cv. Cabernet Sauvigon não afetou os valores de $\mathrm{pH}$, acidez total e SS no mosto das uvas. Além disso, os valores destas variáveis foram muitos próximos aos encontrados no mosto da cv. Cabernet Sauvignon, em experimentos realizados por Rizzon e Miele (2002), que, por exemplo, relatam valor médio de SS de $18,1^{\circ}$ Brix, bem como por Rizzon e Sganzerla (2007), em estudos também realizados na região da Serra Gaúcha do Rio Grande do Sul, mesma região do presente trabalho. No entanto, convém ressaltar que, em solos com textura arenosa superficial e com baixo teor de matéria orgânica e, por consequência, com baixa fertilidade natural, é muito mais provável que o modo de distribuição do composto orgânico possa afetar a composição do mosto.

TABELA 1- Atributos físicos e químicos, na camada de 0-20 cm, de um solo Neossolo Litólico antes da implantação do experimento (Bento Gonçalves-RS).

\begin{tabular}{|c|c|}
\hline Atributo & $0-20 \mathrm{~cm}$ \\
\hline $\operatorname{Argila}^{(1)}\left(\mathrm{g} \mathrm{kg}^{-1}\right)$ & 220 \\
\hline Matéria orgânica ${ }^{(2)}\left(\mathrm{g} \mathrm{kg}^{-1}\right)$ & 29,0 \\
\hline $\mathrm{pH}-\mathrm{H}_{2} \mathrm{O}^{(2)}$ & 5,9 \\
\hline Índice $\mathrm{SMP}^{(2)}$ & 6,4 \\
\hline $\mathrm{P}$ disponível ${ }^{(3)}\left(\mathrm{mg} \mathrm{kg}^{-1}\right)$ & 14,2 \\
\hline $\mathrm{K}$ trocável ${ }^{(3)}\left(\mathrm{mg} \mathrm{kg}^{-1}\right)$ & 145 \\
\hline Ca trocável ${ }^{(4)}\left(\mathrm{cmol}_{\mathrm{c}} \mathrm{kg}^{-1}\right)$ & 9,2 \\
\hline $\operatorname{Mg}$ trocável $^{(4)}\left(\mathrm{cmol}_{\mathrm{c}} \mathrm{kg}^{-1}\right)$ & 1,8 \\
\hline Al trocável ${ }^{(4)}\left(\mathrm{cmol}_{\mathrm{c}} \mathrm{kg}^{-1}\right)$ & 0,0 \\
\hline $\mathrm{CTC}_{\mathrm{pH} 7.0}{ }^{(5)}\left(\mathrm{cmol}_{\mathrm{c}} \mathrm{kg}^{-1}\right)$ & 17,7 \\
\hline
\end{tabular}

(1) Método da pipeta (EMBRAPA, 1997); (2) Determinado conforme Tedesco et al. (1995); ${ }^{(3)}$ Extraído por Mehlich 1 (TEDESCO et al., 1995); ${ }^{(4)}$ Extraído por $\mathrm{KCl} 1 \mathrm{~mol} \mathrm{~L}^{-1}$ (TEDESCO et al., 1995); ${ }^{(5)} \mathrm{CTC}_{\mathrm{pH} 7.0}=\mathrm{H}+\mathrm{Al}+\left(\mathrm{Ca}^{2}+\mathrm{Mg}^{2}+\mathrm{K}^{+}\right)$.

TABELA 2- Precipitação pluviométrica e temperatura do ar durante os meses de condução do experimento (Bento Gonçalves-RS).

\begin{tabular}{|c|c|c|c|c|c|c|c|c|c|c|}
\hline \multirow{2}{*}{ Mês } & $\begin{array}{l}\mathrm{PP}^{(1)} \\
(\mathrm{mm})\end{array}$ & $\mathrm{T}\left(\mathrm{C}^{\mathrm{o}}\right)$ & $\begin{array}{l}\mathrm{PP}^{(1)} \\
(\mathrm{mm}) \\
\end{array}$ & $\mathrm{T}\left(\mathrm{C}^{\mathrm{o}}\right)$ & $\begin{array}{l}\mathrm{PP}^{(1)} \\
(\mathrm{mm})\end{array}$ & $\begin{array}{c}\mathrm{T} \\
\left(\mathrm{C}^{\circ}\right) \\
\end{array}$ & $\begin{array}{l}\mathrm{PP}^{(1)} \\
(\mathrm{mm})\end{array}$ & $\mathrm{T}\left(\mathrm{C}^{\mathrm{o}}\right)$ & $\begin{array}{l}\mathrm{PP}^{(1)} \\
(\mathrm{mm})\end{array}$ & $\mathrm{T}\left(\mathrm{C}^{\mathrm{o}}\right)$ \\
\hline & \multicolumn{2}{|c|}{2006} & \multicolumn{2}{|c|}{2007} & \multicolumn{2}{|c|}{2008} & \multicolumn{2}{|c|}{2009} & \multicolumn{2}{|c|}{ Normal 1961 a 1990} \\
\hline Janeiro & 112,6 & 22,8 & 135,7 & 22,3 & 44,8 & 21,3 & 269,6 & 20,4 & 140 & 21,8 \\
\hline Fevereiro & 72,1 & 21,4 & 153,6 & 21,9 & 76,8 & 21,0 & 144,5 & 21,7 & 139 & 21,7 \\
\hline Março & 148 & 21,1 & 208,3 & 22,1 & 91,1 & 20,6 & 90,6 & 21,0 & 128 & 20,3 \\
\hline Abril & 57,6 & 17,2 & 56,6 & 19,4 & 87,4 & 17,1 & 24,2 & 18,4 & 114 & 17,5 \\
\hline Maio & 88,5 & 12,8 & 178,5 & 12,5 & 168,1 & 14,3 & 134,7 & 15,6 & 107 & 14,5 \\
\hline Junho & 199,1 & 14,0 & 60,7 & 13,6 & 160,2 & 11,4 & 82,9 & 11,2 & 157 & 12,8 \\
\hline Julho & 205,4 & 15,0 & 280,5 & 10,5 & 73 & 14,9 & 97,8 & 10,2 & 161 & 12,9 \\
\hline Agosto & 90,7 & 14,3 & 123,4 & 12,9 & 198,5 & 14,1 & 257,9 & 15,2 & 165 & 13,6 \\
\hline Setembro & 106,2 & 14,3 & 270 & 17,7 & 144,1 & 13,2 & 411,7 & 14,6 & 185 & 14,9 \\
\hline Outubro & 56 & 18,7 & 119,2 & 18,6 & 309,6 & 16,8 & 145,1 & 16,7 & 156 & 17,0 \\
\hline Novembro & 154,7 & 18,5 & 162,3 & 17,9 & 70,3 & 19,4 & 359,5 & 21,6 & 140 & 18,9 \\
\hline Dezembro & 66,7 & 23,0 & 209,6 & 21,0 & 85,8 & 20,3 & 232,6 & 21,2 & 144 & 20,7 \\
\hline
\end{tabular}

(1) Precipitação Pluviométrica. ${ }^{(2)}$ Temperatura. Fonte: Embrapa Uva e Vinho (2011). 
TABELA 3- Características químicas médias do composto orgânico aplicado no solo Neossolo Litólico e cultivado com videiras cv. Cabernet Sauvignon (Bento Gonçalves-RS).

\begin{tabular}{lc}
\hline \multicolumn{1}{c}{ Característica(1) } & Valor \\
\hline Carbono orgânico total (\%) & 27,0 \\
Nitrogênio total (\%) & 1,7 \\
Fósforo total (\%) & 0,2 \\
Potássio total (\%) & 1,7 \\
Cálcio total (\%) & 8,2 \\
Magnésio total (\%) & 0,3 \\
Cobre total $\left(\mathrm{mg} \mathrm{kg}^{-1}\right)$ & 18,7 \\
Sódio total $\left(\mathrm{mg} \mathrm{kg}^{-1}\right)$ & 2794,9 \\
Zinco total $\left(\mathrm{mg} \mathrm{kg}^{-1}\right)$ & 38,4 \\
Manganês total $\left(\mathrm{mg} \mathrm{kg}^{-1}\right)$ & 311,2 \\
Ferro total $\left(\mathrm{mg} \mathrm{kg}^{-1}\right)$ & 3616,4 \\
Boro total $\left(\mathrm{mg} \mathrm{kg}^{-1}\right)$ & 15,8 \\
Relação C/N & 15,7 \\
pH em água & 7,4 \\
Umidade (\%) & 40,2 \\
\hline
\end{tabular}

${ }^{(1)}$ Determinado conforme Tedesco et al. (1995).

TABELA 4- Matéria seca da poda hibernal e verde, peso médio e número de cachos, e produção de uva de videiras cv. Cabernet Sauvignon submetidas a diferentes modos de distribuição de composto orgânico (Bento Gonçalves-RS).

\begin{tabular}{|c|c|c|c|}
\hline \multirow{2}{*}{ Tratamento } & \multicolumn{3}{|c|}{ Safra } \\
\hline & $2006 / 2007$ & $2007 / 2008$ & $2008 / 2009$ \\
\hline \multicolumn{4}{|c|}{-----------Matéria seca poda hibernal (g planta ${ }^{-1}$ )----------- } \\
\hline $\mathrm{T} 1^{(1)}$ & $35 c^{(2)}$ & $255 \mathrm{c}$ & $240 \mathrm{~d}$ \\
\hline $\mathrm{T} 2$ & $135 b$ & $340 \mathrm{~b}$ & $324 \mathrm{c}$ \\
\hline $\mathrm{T} 3$ & $100 \mathrm{~b}$ & $345 b$ & $388 \mathrm{a}$ \\
\hline T4 & $30 c$ & $516 a$ & $353 \mathrm{~b}$ \\
\hline T5 & $528 \mathrm{a}$ & $538 \mathrm{a}$ & $356 b$ \\
\hline \multirow[t]{2}{*}{ CV\% } & 25,1 & 14,5 & 14,6 \\
\hline & \multicolumn{3}{|c|}{--------------Matéria seca da poda verde $\left(\right.$ g planta $\left.^{-1}\right)--------$} \\
\hline $\mathrm{T} 1^{(1)}$ & $16 \mathrm{a}$ & $19 a$ & $1000 d$ \\
\hline T2 & $22 \mathrm{a}$ & $26 a$ & $1350 \mathrm{c}$ \\
\hline $\mathrm{T} 3$ & $19 a$ & $25 \mathrm{a}$ & $1620 \mathrm{a}$ \\
\hline $\mathrm{T} 4$ & $21 a$ & $24 a$ & $1473 b$ \\
\hline T5 & $20 a$ & $25 a$ & $1483 b$ \\
\hline \multirow{2}{*}{ CV\% } & 33,2 & 16,2 & 14,80 \\
\hline & \multicolumn{3}{|c|}{------------------Peso médio de cacho (g planta-1)--------- } \\
\hline $\mathrm{T} 1^{(1)}$ & $60,40 \mathrm{a}$ & $53,25 \mathrm{a}$ & $62,10 \mathrm{a}$ \\
\hline T2 & $67.17 \mathrm{a}$ & $58.00 \mathrm{a}$ & $67.00 \mathrm{a}$ \\
\hline $\mathrm{T} 3$ & $58,50 a$ & $56,35 \mathrm{a}$ & $68,01 \mathrm{a}$ \\
\hline T4 & $62,77 \mathrm{a}$ & $58,99 a$ & $72,60 \mathrm{a}$ \\
\hline T5 & $67,93 a$ & $60,88 \mathrm{a}$ & $60,30 \mathrm{a}$ \\
\hline \multirow[t]{2}{*}{$\mathrm{CV} \%$} & 18,7 & 15,8 & 15,90 \\
\hline & \multicolumn{3}{|c|}{ 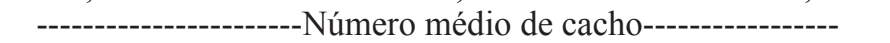 } \\
\hline $\mathrm{T} 1^{(1)}$ & $10 \mathrm{ab}$ & $14 \mathrm{a}$ & $15 \mathrm{a}$ \\
\hline $\mathrm{T} 2$ & $24 a$ & $12 \mathrm{a}$ & $23 a$ \\
\hline $\mathrm{T} 3$ & $19 a$ & $16 a$ & $22 \mathrm{a}$ \\
\hline T4 & $8 b$ & $11 \mathrm{a}$ & $20 \mathrm{a}$ \\
\hline T5 & $14 \mathrm{ab}$ & $18 \mathrm{a}$ & $21 \mathrm{a}$ \\
\hline \multirow[t]{2}{*}{ CV\% } & 30,1 & 15,4 & 25,80 \\
\hline & -------- & $\left.\mathrm{Mg} \mathrm{ha}^{-1}\right)---$ & \\
\hline $\mathrm{T} 1^{(1)}$ & $2,01 \mathrm{a}$ & $2,48 \mathrm{a}$ & $3,10 \mathrm{a}$ \\
\hline T2 & $0,90 \mathrm{a}$ & $2,31 \mathrm{a}$ & $5,14 a$ \\
\hline T3 & $3,70 \mathrm{a}$ & $3,00 \mathrm{a}$ & $4,98 \mathrm{a}$ \\
\hline $\mathrm{T} 4$ & $1,67 \mathrm{a}$ & $2,16 a$ & $4,84 a$ \\
\hline T5 & $3,16 a$ & $3,65 a$ & $4,22 \mathrm{a}$ \\
\hline CV\% & 29,02 & 25,20 & 27,5 \\
\hline
\end{tabular}

(1) T1- Testemunha; T2- aplicação anual de $20 \mathrm{~m}^{3}$ ha-1 de composto orgânico, 15 dias antes da poda, em faixa de 30 x $60 \mathrm{~cm}$, na superfície do solo (aproximadamente a $30 \mathrm{~cm}$ do caule da planta) e sempre do mesmo lado da linha de plantio; T3- Ídem ao T2, mas o composto foi aplicado anualmente em lados alternados da linha de plantio das plantas; T4- Ídem ao T2, mas o composto foi distribuído em covas (dimensões 20 x 60 x $20 \mathrm{~cm}$ ) na linha de plantio, sempre do mesmo lado das plantas, e T5- Ídem ao T2, mas o composto orgânico foi aplicado em covas (dimensões $20 \times 60 \times 20 \mathrm{~cm}$ ) e com distribuição anual em lados alternados da linha de plantio. ${ }^{(2)}$ Médias seguidas por letras iguais, na coluna, não diferem entre si, pelo teste de Tukey, a 5\% de probabilidade. 
TABELA 5- Teores totais de nitrogênio, fósforo, potássio, cálcio, magnésio e boro em folhas completas de videiras cv. Cabernet Sauvignon submetidas a diferentes modos de distribuição de composto orgânico (Bento Gonçalves-RS).

\begin{tabular}{|c|c|c|c|}
\hline \multirow{2}{*}{ Tratamento } & \multicolumn{3}{|c|}{ Safra } \\
\hline & $2006 / 2007$ & $2007 / 2008$ & $2008 / 2009$ \\
\hline & - & rogênio ( $\mathrm{g} \mathrm{k}$ & \\
\hline $\mathrm{T} 1^{(1)}$ & $2,12 \mathrm{a}^{(2)}$ & $2,02 \mathrm{a}$ & $2,40 \mathrm{a}$ \\
\hline $\mathrm{T} 2$ & $2,18 \mathrm{a}$ & $2,11 \mathrm{a}$ & $2,65 a$ \\
\hline T3 & $2,10 \mathrm{a}$ & $2,15 \mathrm{a}$ & $2,47 \mathrm{a}$ \\
\hline $\mathrm{T} 4$ & $2,20 \mathrm{a}$ & $2,05 \mathrm{a}$ & $2,38 \mathrm{a}$ \\
\hline T5 & $2,36 \mathrm{a}$ & $2,20 \mathrm{a}$ & $2,62 \mathrm{a}$ \\
\hline CV\% & 11,4 & 12,5 & 10,6 \\
\hline T1 & $0,14 \mathrm{a}$ & $0,25 \mathrm{a}^{\circ}$ & $0,37 \mathrm{a}$ \\
\hline $\mathrm{T} 2$ & $0,14 \mathrm{a}$ & $0,26 a$ & $0,42 \mathrm{a}$ \\
\hline T3 & $0,17 \mathrm{a}$ & $0,25 \mathrm{a}$ & $0,43 \mathrm{a}$ \\
\hline T4 & $0,21 \mathrm{a}$ & $0,28 \mathrm{a}$ & $0,48 \mathrm{a}$ \\
\hline T5 & $0,31 \mathrm{a}$ & $0,28 \mathrm{a}$ & $0,43 \mathrm{a}$ \\
\hline CV\% & 19,6 & 17,5 & 12,3 \\
\hline T1 & $1,32 \mathrm{ab}$ & $1,45 \mathrm{a}$ & $1,35 \mathrm{a}$ \\
\hline $\mathrm{T} 2$ & 1,09abc & $1,05 \mathrm{a}$ & $1,22 \mathrm{a}$ \\
\hline T3 & $0,76 \mathrm{c}$ & $1,21 \mathrm{a}$ & $1,06 \mathrm{a}$ \\
\hline $\mathrm{T} 4$ & $1,40 \mathrm{a}$ & $1,65 \mathrm{a}$ & $1,05 \mathrm{a}$ \\
\hline $\mathrm{T} 5$ & $1,02 \mathrm{abc}$ & $1,34 \mathrm{a}$ & $0,97 \mathrm{a}$ \\
\hline $\mathrm{CV} \%$ & 12,1 & 11,3 & 12,1 \\
\hline T1 & $1,67 b c$ & $1,69 \mathrm{a}$ & $1,09 \mathrm{a}$ \\
\hline $\mathrm{T} 2$ & $1,62 b c$ & $1,77 \mathrm{a}$ & $1,02 \mathrm{a}$ \\
\hline T3 & $1,80 \mathrm{a}$ & $1,99 a$ & $1,32 \mathrm{a}$ \\
\hline $\mathrm{T} 4$ & $1,61 b c$ & $1,72 \mathrm{a}$ & $1,22 \mathrm{a}$ \\
\hline T5 & $1,72 \mathrm{ab}$ & $1,97 \mathrm{a}$ & $1,19 \mathrm{a}$ \\
\hline CV\% & 13,6 & 18,1 & 14,1 \\
\hline $\mathrm{T} 1$ & $3,7 \mathrm{a}$ & $1,5 \mathrm{a}$ & $2,2 \mathrm{a}$ \\
\hline $\mathrm{T} 2$ & $3,6 a$ & $1,7 \mathrm{a}$ & $2,3 a$ \\
\hline T3 & $4,5 \mathrm{a}$ & $2,1 \mathrm{a}$ & $2,1 \mathrm{a}$ \\
\hline $\mathrm{T} 4$ & $3,8 \mathrm{a}$ & $2,2 \mathrm{a}$ & $2,5 \mathrm{a}$ \\
\hline T5 & $4,6 a$ & $2,3 \mathrm{a}$ & $2,4 a$ \\
\hline CV\% & 11,0 & 18,2 & 15,0 \\
\hline
\end{tabular}

(1)T1- Testemunha; T2- aplicação anual de $20 \mathrm{~m}^{3} \mathrm{ha}^{-1}$ de composto orgânico, 15 dias antes da poda, em faixa de $30 \mathrm{x} 60 \mathrm{~cm}$, na superfície do solo (aproximadamente a $30 \mathrm{~cm}$ do caule da planta) e sempre do mesmo lado da linha de plantio; T3- Ídem ao T2, mas o composto foi aplicado anualmente em lados alternados da linha de plantio das plantas; T4- Ídem ao T2, mas o composto foi distribuído em covas (dimensões 20 × 60 × $20 \mathrm{~cm}$ ) na linha de plantio, sempre do mesmo lado das plantas, e T5- Ídem ao T2, mas o composto orgânico foi aplicado em covas (dimensões 20 × 60 × $20 \mathrm{~cm}$ ) e com distribuição anual em lados alternados da linha de plantio. ${ }^{(2)}$ Médias seguidas por letras iguais, na coluna, não diferem entre si, pelo teste de Tukey, a $5 \%$ de probabilidade. 
TABELA 6- Valores de sólidos solúveis (SS), pH, acidez titulável, ácido tartárico e málico no mosto de uvas cv. Cabernet Sauvignon submetidas a diferentes modos de distribuição de composto (Bento Gonçalves-RS).

\begin{tabular}{|c|c|c|c|}
\hline \multirow{2}{*}{ Tratamento } & \multicolumn{3}{|c|}{ Safra } \\
\hline & $2006 / 2007$ & $2007 / 2008$ & $2008 / 2009$ \\
\hline & - & ------SS--- & 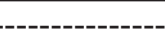 \\
\hline $\mathrm{T} 1^{(1)}$ & $17,8 \mathrm{a}^{(2)}$ & $18,1 \mathrm{a}$ & $18,2 \mathrm{a}$ \\
\hline $\mathrm{T} 2$ & $17,4 \mathrm{a}$ & $17,2 \mathrm{a}$ & $18,0 \mathrm{a}$ \\
\hline $\mathrm{T} 3$ & $17,9 a$ & $17,1 \mathrm{a}$ & $18,1 \mathrm{a}$ \\
\hline $\mathrm{T} 4$ & $17,2 \mathrm{a}$ & $18,3 \mathrm{a}$ & $17,6 a$ \\
\hline T5 & $18,4 \mathrm{a}$ & $17,2 \mathrm{a}$ & $17,5 \mathrm{a}$ \\
\hline $\mathrm{CV} \%$ & 7,1 & 4,3 & 6,4 \\
\hline $\mathrm{T} 1^{(1)}$ & $3,94 a$ & $3,82 \mathrm{a}$ & $3,32 \mathrm{a}$ \\
\hline $\mathrm{T} 2$ & $3,88 \mathrm{a}$ & $3,76 \mathrm{a}$ & $3,81 \mathrm{a}$ \\
\hline T3 & $3,88 \mathrm{a}$ & $3,84 \mathrm{a}$ & $3,76 a$ \\
\hline $\mathrm{T} 4$ & $3,81 \mathrm{a}$ & $3,96 \mathrm{a}$ & $3,88 \mathrm{a}$ \\
\hline $\mathrm{T} 5$ & $3,74 \mathrm{a}$ & $3,62 \mathrm{a}$ & $3,72 \mathrm{a}$ \\
\hline $\mathrm{CV} \%$ & 4,8 & 5,2 & 7,20 \\
\hline $\mathrm{T} 1^{(1)}$ & $64,08 \mathrm{a}$ & $\begin{array}{l}\text { z titulável (n } \\
60,32 \mathrm{a}\end{array}$ & $59,22 \mathrm{a}$ \\
\hline $\mathrm{T} 2$ & $59,56 \mathrm{a}$ & $62,44 a$ & $58,32 \mathrm{a}$ \\
\hline $\mathrm{T} 3$ & $63,28 \mathrm{a}$ & $61,42 \mathrm{a}$ & $60,88 \mathrm{a}$ \\
\hline $\mathrm{T} 4$ & $65,11 \mathrm{a}$ & $59,28 \mathrm{a}$ & $61,44 a$ \\
\hline $\mathrm{T} 5$ & $61,44 a$ & $64,08 \mathrm{a}$ & $64,28 \mathrm{a}$ \\
\hline $\mathrm{CV} \%$ & 7,8 & 12,4 & 11,3 \\
\hline $\mathrm{T} 1^{(1)}$ & $6,8 \mathrm{a}$ & $\begin{array}{c}\text { artático (g L- } \\
7,2 \mathrm{a}\end{array}$ & $7,2 \mathrm{a}$ \\
\hline $\mathrm{T} 2$ & $6,8 \mathrm{a}$ & $6,9 a$ & $6,4 a$ \\
\hline $\mathrm{T} 3$ & $6,8 \mathrm{a}$ & $6,8 \mathrm{a}$ & $7,4 a$ \\
\hline $\mathrm{T} 4$ & $7,7 \mathrm{a}$ & $6,9 a$ & $7,9 a$ \\
\hline T5 & $6,7 a$ & $6,8 \mathrm{a}$ & $7,1 \mathrm{a}$ \\
\hline $\mathrm{CV} \%$ & 7,8 & 6,9 & 10,1 \\
\hline $\mathrm{T} 1^{(1)}$ & $5,8 \mathrm{a}$ & $\begin{array}{c}\text { lo Málico (g } \\
5,9 \mathrm{a}\end{array}$ & $6,2 a$ \\
\hline $\mathrm{T} 2$ & $6,2 a$ & $6,2 a$ & $5,9 \mathrm{a}$ \\
\hline T3 & $6,1 \mathrm{a}$ & $5,9 a$ & $6,2 a$ \\
\hline $\mathrm{T} 4$ & $6,2 \mathrm{a}$ & $5,9 \mathrm{a}$ & $6,1 \mathrm{a}$ \\
\hline $\mathrm{T} 5$ & $5,9 a$ & $6,0 \mathrm{a}$ & $6,2 \mathrm{a}$ \\
\hline $\mathrm{CV} \%$ & 7,5 & 10,1 & 9,8 \\
\hline
\end{tabular}

${ }^{(1)}$ T1- Testemunha; T2- aplicação anual de $20 \mathrm{~m}^{3} \mathrm{ha}^{-1}$ de composto orgânico, 15 dias antes da poda, em faixa de $30 \mathrm{x} 60 \mathrm{~cm}$, na superfície do solo (aproximadamente a $30 \mathrm{~cm}$ do caule da planta) e sempre do mesmo lado da linha de plantio; T3- Ídem ao T2, mas o composto foi aplicado anualmente em lados alternados da linha de plantio das plantas; T4- Ídem ao T2, mas o composto foi distribuído em covas (dimensões $20 \times 60 \times 20 \mathrm{~cm}$ ) na linha de plantio, sempre do mesmo lado das plantas, e T5- Ídem ao T2, mas o composto orgânico foi aplicado em covas (dimensões 20 × 60 × $20 \mathrm{~cm}$ ) e com distribuição anual em lados alternados da linha de plantio. ${ }^{(2)}$ Médias seguidas por letras iguais, na coluna, não diferem entre si, pelo teste de Tukey, a 5\% de probabilidade. 


\section{CONCLUSÕES}

1- A distribuição de composto orgânico em covas sempre do mesmo lado da linha de plantio ou em lados alternados, ao longo dos anos, aumenta a quantidade de matéria seca dos ramos podados no inverno, especialmente nas primeiras safras depois de sua aplicação.

2- A aplicação e o modo de distribuição do composto orgânico na superfície do solo ou incorporado, anualmente no mesmo lado da linha de plantio ou alternado entre os lados ao longo dos anos, não afeta a produção de uvas, bem como não altera o estado nutricional das plantas nem a composição do mosto.

3- O composto orgânico deve ser aplicado na superfície do solo sem incorporação, em um lado da linha de plantio ou em lados alternados ao longo dos anos.

\section{REFERENCIAS}

AUGUSTE, M. H. Application de la chomatographie enphase liquide à haute pression à l'analyse des moûts etdes vins. 1979. 135f. Tese (Doutorado em Enologia-Ampelologia) - Université de Bordeaux II, Bordeaux, 1979.

BERTRAN, E.; SORT, X.; SOLIVA, M.; TRILLAS, I. Composting winery waste: sludges and grape stalks. Bioresource Technology, Essex, v.95, p.203-208, 2004.

BRUNETTO, G. Nitrogênio em videira: Recuperação, acumulação e alterações na produtividade e na composição da uva. 2008. 163f. Tese (Doutorado em Ciência do Solo) - Universidade Federal de Santa Maria, Santa Maria, 2008.

BRUNETTO, G.; CERETTA, C.A.; KAMINSKI, J; MELO, G.W.B; GIROTTO, E.; TRENTIN, E.E.; LOURENZI, C.R.; VIEIRA, R.C.B.; GATIBONI, L.C. Produção e composição química da uva em videiras submetidas à adubação nitrogenada na Serra Gaúcha do Rio Grande do Sul. Ciência Rural, Santa Maria, v.39, p.2035-2041, 2009.
BRUNETTO, G.; GIROTTO, E.; MELO, G.W.B.; SANTOS, D. R.; CERETTA, C.A.; KAMINSKI, J.; VIEIRA, R.C.B. Aplicação foliar de nitrogênio em videira: Avaliação do teor na folha e das reservas nitrogenadas e de carboidratos nas gemas dos ramos do ano. Revista Brasileira de Fruticultura, Jaboticabal, v.30, p.1119-1123, 2008.

BRUNETTO, G.; KAMINSKI, J.; MELO, G.W.B.; BRUNING, F.S.; MALLMANN, F.J.K. Destino do nitrogênio em videiras 'Chardonnay' e 'Riesling Renano' quando aplicado no inchamento das gemas.

Revista Brasileira de Fruticultura, Jaboticabal, v.28, p.497-500, 2006.

BRUNETTO, G.; MELO, G.W.; KAMINSKI, J.; CERETTA, C. A. Adubação nitrogenada em ciclos consecutivos e seu impacto na produção e na qualidade do pêssego. Pesquisa Agropecuária Brasileira, Brasília, v.42, n.12., p. 1721-1725, 2007.

BRUNETTO, G.; CERETTA, C.A.; KAMINSKI, J; MELO, G.W.B; GIROTTO, E.; TRENTIN, E.E.; LOURENZI, C.R.; VIEIRA, R.C.B.; GATIBONI, L.C. Produção e composição química da uva em videiras submetidas à adubação nitrogenada na Serra Gaúcha do Rio Grande do Sul. Ciência Rural, Santa Maria, v.39, p.2035-2041, 2009.

BRUNETTO, G.; GIROTTO, E.; MELO, G.W.B.; SANTOS, D. R.; CERETTA, C.A.; KAMINSKI, J.; VIEIRA, R.C.B. Aplicação foliar de nitrogênio em videira: Avaliação do teor na folha e das reservas nitrogenadas e de carboidratos nas gemas dos ramos do ano. Revista Brasileira de Fruticultura, Jaboticabal, v.30, p.1119-1123, 2008.

BRUNETTO, G.; VENTURA, M.; SCANDELLARI, F.; CERETTA, C.A.; KAMINSKI, J.; MELO, G.W.B.; TAGLIAVINI, M. Nutrients release during the decomposition of mowed perennial ryegrass and white clover and its contribution to nitrogen nutrition of grapevine. Nutrient Cycling in Agroecosystems, Dordrecht, v.90, n.3, p.299-308, 2011.

BUSTAMANTE, M.A.; SAIL-PULLICINO, D.; AGUlLÓ, E.; ANDREU, J.; PAREDES, C.; MORAL, R. Application of winneryand distilleywast compost to a Jumilla(SE Spain) vineyard: Effects on the characteristics of a calcareous Sandy-loam soil. Agriculture, Ecosystems and Environment, Amsterdam, v.140, p.80-87, 2011. 
CHISTOU, M.; AVRAMIDES, E.J.; JONES, D.L. Dissolved organic nitrogen dynamics in a Mediterranean vineyard soil. Soil Biology \& Biochemistry, Elmsford, v.38, p.2265-2277, 2006.

CQFS - Comissão de Química e Fertilidade do Solo - RS/SC. Manual de adubação e calagem para os Estados do Rio Grande do Sul e de Santa Catarina. 10.ed. Porto Alegre: SBCS - Núcleo Regional Sul/UFRGS, 2004. 400p.

EMBRAPA-CNPS-CNPS. Manual de métodos de análise de solos. Brasília. EMBRAPA. Rio de Janeiro, 1997. 212 p.

EMBRAPA-CNPS. Sistema brasileiro de classificação de solos. 2.ed. Brasília. EMBRAPA, 2006. $306 \mathrm{p}$.

EMBRAPA-UVA E VINHO. Dados meteorológicos - estação agroclimática da Embrapa Uva e Vinho. Disponível em: <http://www.cnpuv.embrapa.br/ prodserv/meteorologia>. Acesso em: 04 maio 2011.

GOULET, E.; DOUSSET, S.; CHAUSSOD, R.; BARTOLI, F.; DOLÉDEC, A.F.; ANDREUX, F.; Water-stable aggregates and organic matter pools in a calcareous vineyard soil under four soil-surface management systems. Soil Use Management, Oxford, v.20, p.318-324, 2004.

KORBOULEWSKY, N.; DUPOUYET, S.; BONIN, $\mathrm{G}$. Environmental risks of applying sewage sludge compost to vineyards: carbon, heavy metals, nitrogen and phosphorus accumulation. Journal Environmental Quality, Madison, v.31, p.1522-1527, 2002.

MANDELLI, F.; MIELE, A. Uvas americanas e híbridas para processamento em clima temperadopoda. Disponível em: < $\underline{\text { http://sistemasdeproducao. }}$ cnptia.embrapa.br/FontesHTML/Uva/UvaAmerica-

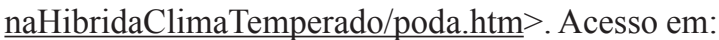
15 jan. 2008.

MORLAT, R. Long-term additions of organic amendments in a Loire Valley vineyard on a calcareous sandy soil. II. Effects on root system, growth, grape yield and foliar nutrient status of a Cabernet franc vine. American Journal Enology and Viticulture, Davis, v.59, n.4, p.364-374, 2008.
MORLAT, R.; SYMONEAUX, R. Long-Term Additions of Organic Amendments in a Loire Valley Vineyard on a Calcareous Sandy Soil. III. Effects on Fruit Composition and Chemical and Sensory Characteristics of Cabernet franc Wine American Journal Enology and Viticulture, Davis, v.59, n.4, p.375-386, 2008.

NASH, D.; BUTLER, C.; CODY, J.; WARNE, M.ST.J.; MCLAUGHLIN, M.J; HEEMSBERGEN, D.; BROOS, K; BELL, M.; BARRY, G.; PRITCHARD, D.; PENNY, N. Effects of biosolids application on pasture and grape vines in SouthEastern Australia. Applied and Environmental Soil Science, 2011. Disponível em: <http://www.doi.org/ $10.1155 / 2011 / 342916>$

NENDEL, C.; KERSEBAUM, K.C.; NIEDER, R.; KUBIAK, R. Nitrogen mineralization from mature bio-waste compost in vineyard soils. III Simulation of soil mineral-nitrogen dynamics. Journal of Plant Nutrition and Soil Science, Malden, v.170, p.598-607, 2007.

PINAMONTI, F. Compost mulch effects on soil fertility, nutritional status and performance of grapevine. Nutrient Cycling in Agroecosystems, Dordrecht, v.51, p. 239-248, 1998.

PINAMONTI, F.; ZORZI, G.; GASPERI, F.; SILVESTRI, S.; STRINGARI, G. Growth and nutritional status of apples trees and grapevines in municipal solid-waste-amended soil. Acta Horticulturae, Wageningen., v.383, p. 313-321, 1995.

RAMOS, M.C.; MARTINÉZ-CASANOVAS, J.A Nutrient losses by runoff in vineyards of the Mediterranean Alt Penedès region (NE Span). Agriculture, Ecosystems and Environment, Amsterdam, v.113, p.352-363, 2006.

RIZZON, L. A.; MIELE, A. Acidez na vinificação em tinto das uvas Isabel, Cabernet Sauvignon e Cabernet Franc. Ciência Rural, Santa Maria, v.32, n.3, p.511-515, 2002.

RIZZON, L.A.; SGANZERLA, V.M.A. Ácidos tartárico e málico no mosto de uva em Bento Gonçalves-RS. Ciência Rural, Santa Maria, v.37, n.3, p.911-914, 2007. 
SANHUEZA, R.M.V.; SÔNEGO, O.R. Descrição e recomendações de manejo da fusariose da videira (Fusarium oxysporum f. Sp. Herbemonthis). Bento Gonçalves: Embrapa Uva e Vinho, 1993. 4p. (Comunicado Técnico, 12).

SAS Institute Inc ${ }^{\circledR}$ (2008) SAS Ver. 9.1. 3 SAS Institute Inc.: Cary, NC, USA. Lic. Embrapa Uva e Vinho.
SCHENATO, P.G.; MELO, G.W.; SANTOS, H.P.; FIALHO, F.B.; FURLANETTO, V.; BRUNETTO, G.; DORNELES, L.T. Influência do etefon na distribuição de nutrientes e carboidratos e sobre o crescimento em videiras jovens. Revista Brasileira de Fruticultura, Jaboticabal, v.29, n.2, p. 217-221, 2007.

TEDESCO, M. J.; GIANELLO, C.; BISSANI, C. A.; BOHNEN, H.; VOLKWEISS, S. J. Análise de solo, plantas e outros materiais. Porto Alegre: UFRGS, 1995. $174 \mathrm{p}$. 